\title{
RESISTENCIA AL CORTE EN ARCILLA DE SANTIAGO UNDAMEO, MÉXICO CON CAL
}

\author{
O. S. MIRANDA-LEAL ${ }^{1}$, E. A. NÚÑEZ-GUZMÁN¹, W. MARTÍNEZ-MOLINA², \\ C. B. REYES-MORALES ${ }^{3}$, M. A. MONDRAGÓN-ORNELAS ${ }^{1}$ \\ ${ }^{1}$ Laboratorio de Materiales “Ing. Luis Silva Ruelas", Facultad de Ingeniería Civil, Universidad Michoacana de San \\ Nicolás de Hidalgo, Morelia, Michoacán, México, 58210, Gral. Francisco J Mujica. \\ ${ }^{2}$ Facultad de Ingeniería Civil, Universidad Michoacana de San Nicolás de Hidalgo, Morelia, Michoacán, \\ México, 58210, Gral. Francisco J Mujica. \\ ${ }^{3}$ Doctorado en Ingeniería Química, Facultad de Ingeniería Química, Universidad Michoacana de San Nicolás de Hidalgo, \\ Morelia, Michoacán, México, 58210, Gral. Francisco J Mujica.
}

\section{RESUMEN}

Este trabajo presenta y discute los resultados obtenidos de los estudios experimentales realizado para evaluar la estabilización utilizando Cal en arcilla de Santiago Undameo, México. En las proporciones de adición de $3 \%$ de cal con respecto a la masa de arcilla. Se realizaron las siguientes pruebas: granulometría, límites de consistencia para su clasificación de acuerdo con el Sistema Unificado de Clasificación de Suelos (SUCS), Proctor estándar variante A, remoldeada, compresión simple y triaxiales no consolidadas no drenadas (UU). La cal es uno de los estabilizadores de suelo más utilizados en la construcción, la adición 3\% de cal obtuvo una mejora en la resistencia al corte de la arcilla en comparación al testigo, lo cual se ve reflejado en el aumento en la envolvente de falla.

Palabras clave: arcilla; cal; resistencia al corte; cohesión; ángulo de fricción.

\begin{abstract}
This paper presents and discusses the results obtained from the experimental studies carried out to evaluate the stabilization using Cal in clay from Santiago Undameo, Mexico. In the proportions of addition of 3\% lime with respect to the clay mass. The following tests were carried out: granulometry, consistency limits for their classification according to the Unified Soil Classification System (SUCS), standard Proctor variant A, remoulded, simple compression and triaxial unconsolidated undrained (UU). The lime is one of the most used soil stabilizers in the construction, the addition 3\% of lime obtained an improvement in the resistance to the cut of the clay in comparison to the witness, which is reflected in the increase in the envelope of failure.
\end{abstract}

Keywords: clay; lime; the shear resistance; cohesion; angle of friction.

\section{RESUMO}

Este artigo apresenta e discute os resultados obtidos a partir dos estudos experimentais realizados para avaliar a estabilização usando Cal em argila de Santiago Undameo, México. Nas proporções de adição de $3 \%$ de calcário em relação à massa argilosa. Foram realizados os seguintes testes: granulometria, limites de consistência para sua classificação segundo o Sistema Único de Classificação de Solos (SUCS), padrão Proctor variante $\mathrm{A}$, remoldado, simples compressão e triaxial não consolidado não drenado (UU). A cal é um dos estabilizadores de solo mais utilizados na construção, a adição de 3\% de cal obteve uma melhora na resistência ao corte da argila em comparação à testemunha, o que se reflete no aumento do envelope de falha.

Palavras-chave: argila; cal; resistência ao corte; coesão; ângulo de fricção. 


\section{OBJETIVO.}

Se analizará la arcilla de Santiago Undameo, México, a la cual se estabilizará con Cal, con el fin de conocer el aumento en la resistencia al corte y en el ángulo de fricción interna, los cuales se obtendrán de la realización de pruebas triaxiales no consolidadas no drenadas (UU), se realizara las envolventes de falla con la finalidad de analizar el aumento en la resistencia al corte de las muestras realizadas.

\section{METODOLOGÍA}

El suelo se utiliza como material de construcción en diversas obras de ingeniería por eso es de suma importación conocer las propiedades físicas y mecánicas.

La presente investigación está correspondida por el análisis de la arcilla de Santiago Undameo, México. Se realizó una clasificación de suelos realizando: granulométrica (ASTM D422), límites de consistencia (ASTM D 4318-84), para la prueba de resistencia al corte se realiza las pruebas triaxial UU (No consolidadas, No drenadas) (ASTM D2850) a detalle para la arcilla testigo y para la arcilla con Cal.

\section{PRUEBAS DE LABORATORIO}

La prueba granulométrica (ASTM D422) de la arcilla de Santiago Undameo, México. Se realizaron para la clasificación del suelo según SUCS (Sistema Unificado de Clasificación de suelos). Los resultados obtenidos se muestran en la Fig.1.

Para clasificar la arcilla con cemento Portland es necesario realizar la prueba: límites de consistencia (ASTM D 4318-84), esta prueba sirve para clasificación de suelos finos (arcillas y limos), los resultados obtenidos se graficaron en la carta de plasticidad en la Fig. 2.

\section{PRUEBA PROCTOR ESTÁNDAR VARIANTE A}

Para poder obtener el espécimen que se van a someter a la prueba de resistencia al corte (Triaxial UU), se tiene que realizar una remoldeada del material la cual se lleva a cabo aplicando las relaciones volumétricas y gravimétricas (Juárez Badillo, 2005, p. 51).

De la prueba Proctor estándar variante A (ASTM D698), se obtuvieron los siguientes valores de peso específico seco máximo y contenido de humedad óptimo para arcilla testigo y arcilla con Cal, los resultados obtenidos se muestran en la Tabla 1.

\section{PRUEBAS DE RESISTENCIA AL CORTE TRIAXIALES UU (NO CONSOLIDADAS, NO DRENADAS) (ASTM D2850)}

El estabilizador utilizado fue Cal con un 3\% de la masa total. Se obtienen las muestras para las pruebas triaxiales, una vez preparado el espécimen sobre la cámara triaxial se aplica carga en la maquina Soiltest T-500 hasta que llegue a la falla del material en estudio, se repite la prueba con tres confinamientos diferentes $\sigma 3=0.3 \mathrm{~kg} / \mathrm{cm} 2, \sigma 3=0.6 \mathrm{~kg} / \mathrm{cm} 2$ y $\sigma 3=0.9 \mathrm{~kg} / \mathrm{cm} 2$.

Los parámetros más importantes de las pruebas triaxiales UU son: cálculo del esfuerzo y deformación unitaria con tres confinamientos diferentes como se muestra Fig. 3 y Fig. 4.

\section{CRITERIO DE FALLA, MOHR-COULOMB.}

Se grafican los Círculos de Mohr (Braja M. Das, 2001, p. 207). Los cuales se representan en la Fig. 5 y Fig.6. 
Esta teoría afirma que un material falla debido a una combinación crítica de esfuerzo normal y esfuerzo cortante, y no sólo por la presencia de un esfuerzo máximo normal o bien de un esfuerzo máximo cortante (Braja M. Das, 2001, p. 209).

\section{RESULTADOS}

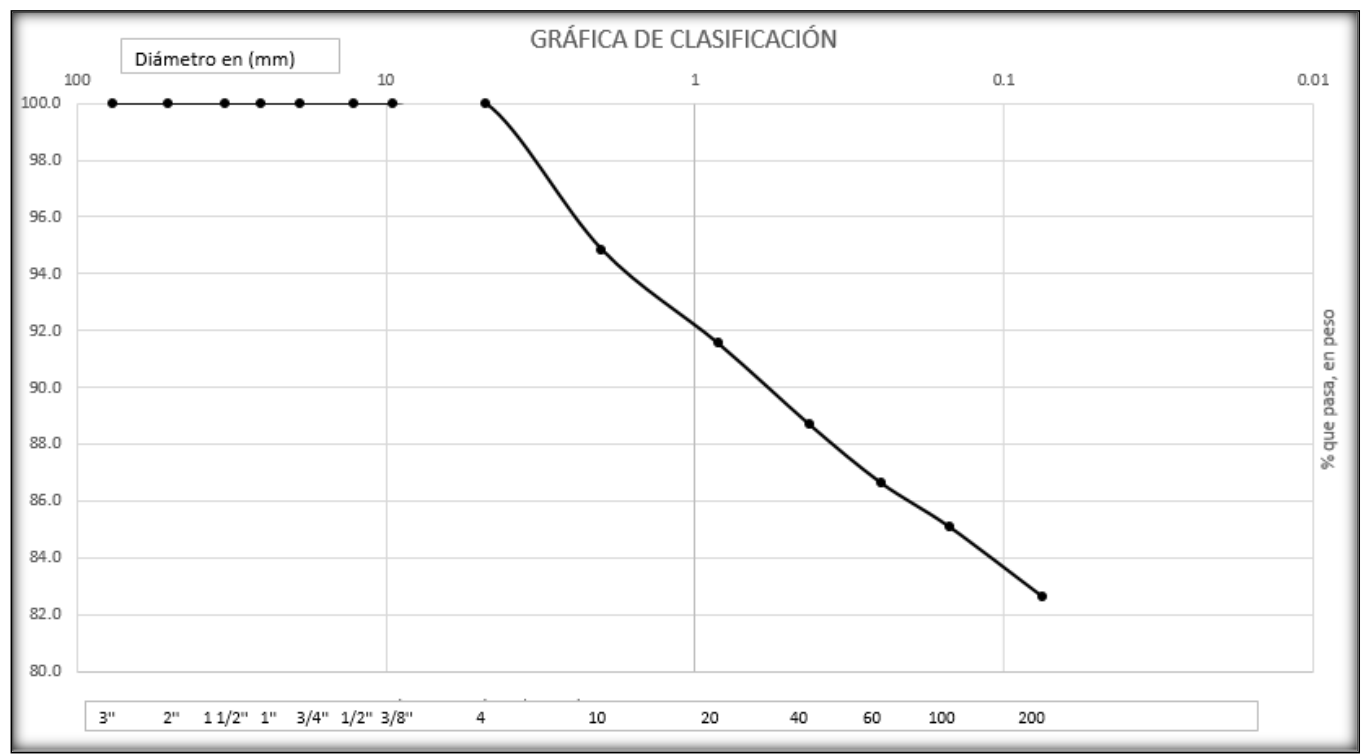

Figura 1. Resultado de ASTM D422, granulometría de la arcilla de Santiago Undameo, México.

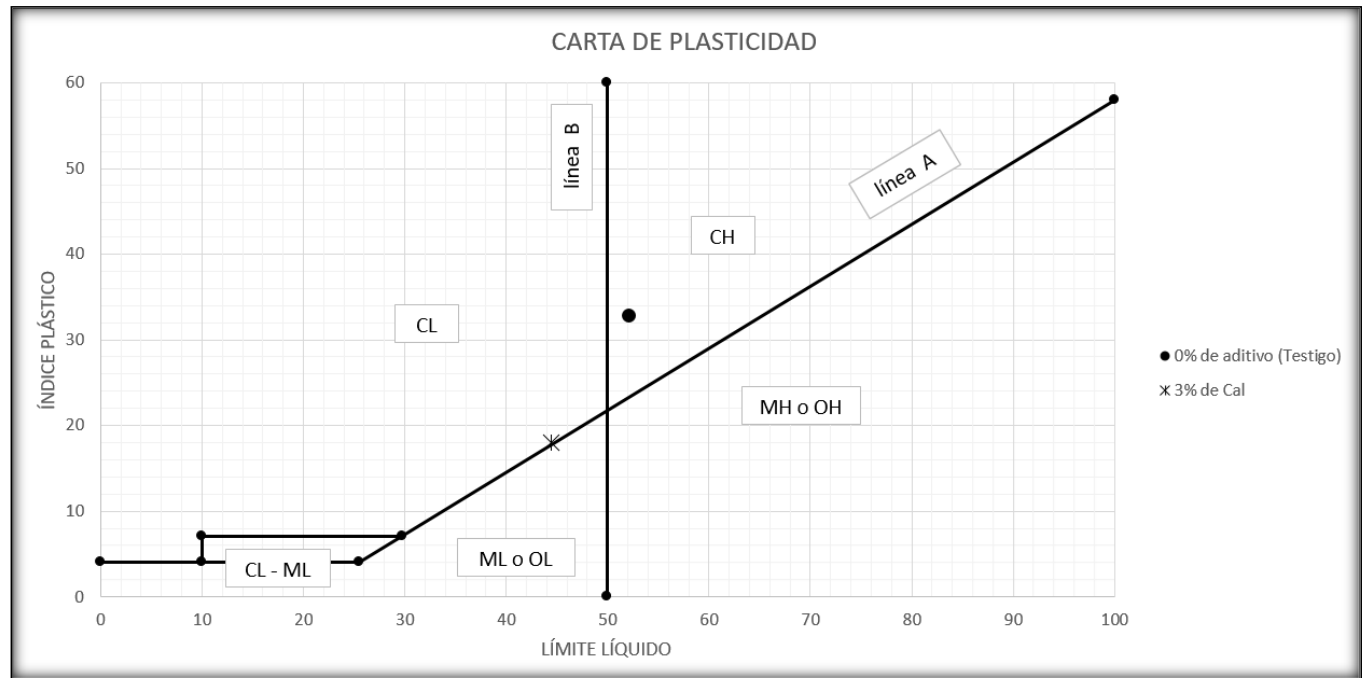

Figura 2. Resultado de límites de consistencia (ASTM D 4318-84) arcilla tesitgo y con Cal de Santiago Undameo, México.

Tabla 1. Resultados de las pruebas Proctor estándar variante A (ASTM D698), de arcilla testigo y arcilla con cemento Portland.

\begin{tabular}{|c|c|c|}
\hline Tipo & Arcilla Testigo & Arcilla con Cal \\
\hline$\left(\mathrm{gr} / \mathrm{cm}^{3}\right)$ & 1.571 & 1.536 \\
\hline $\mathrm{W} \%$ & 26 & 25,6 \\
\hline
\end{tabular}




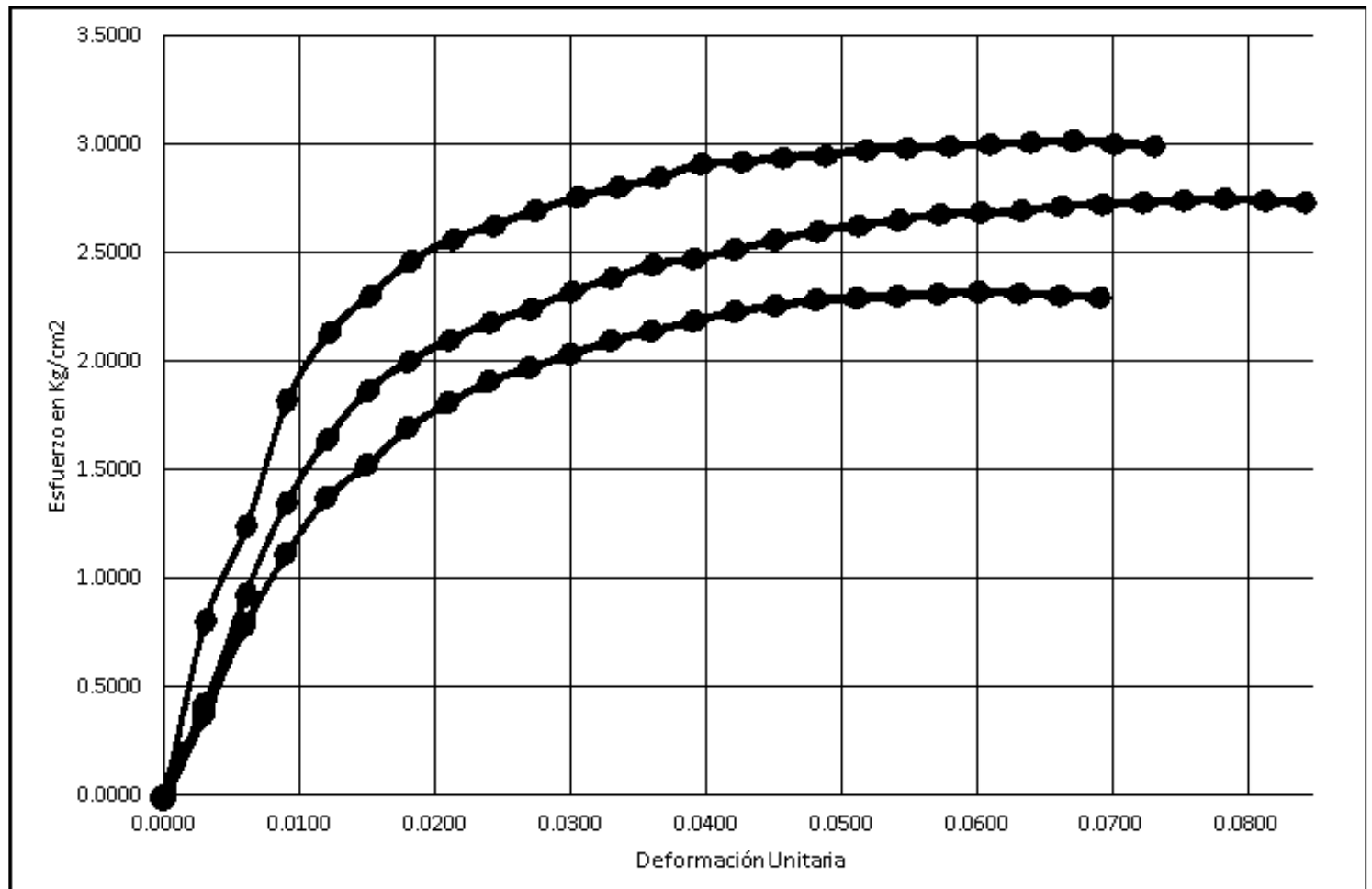

Figura 3. Gráfica esfuerzo- Deformación unitaria de arcilla testigo de Santiago Undameo.

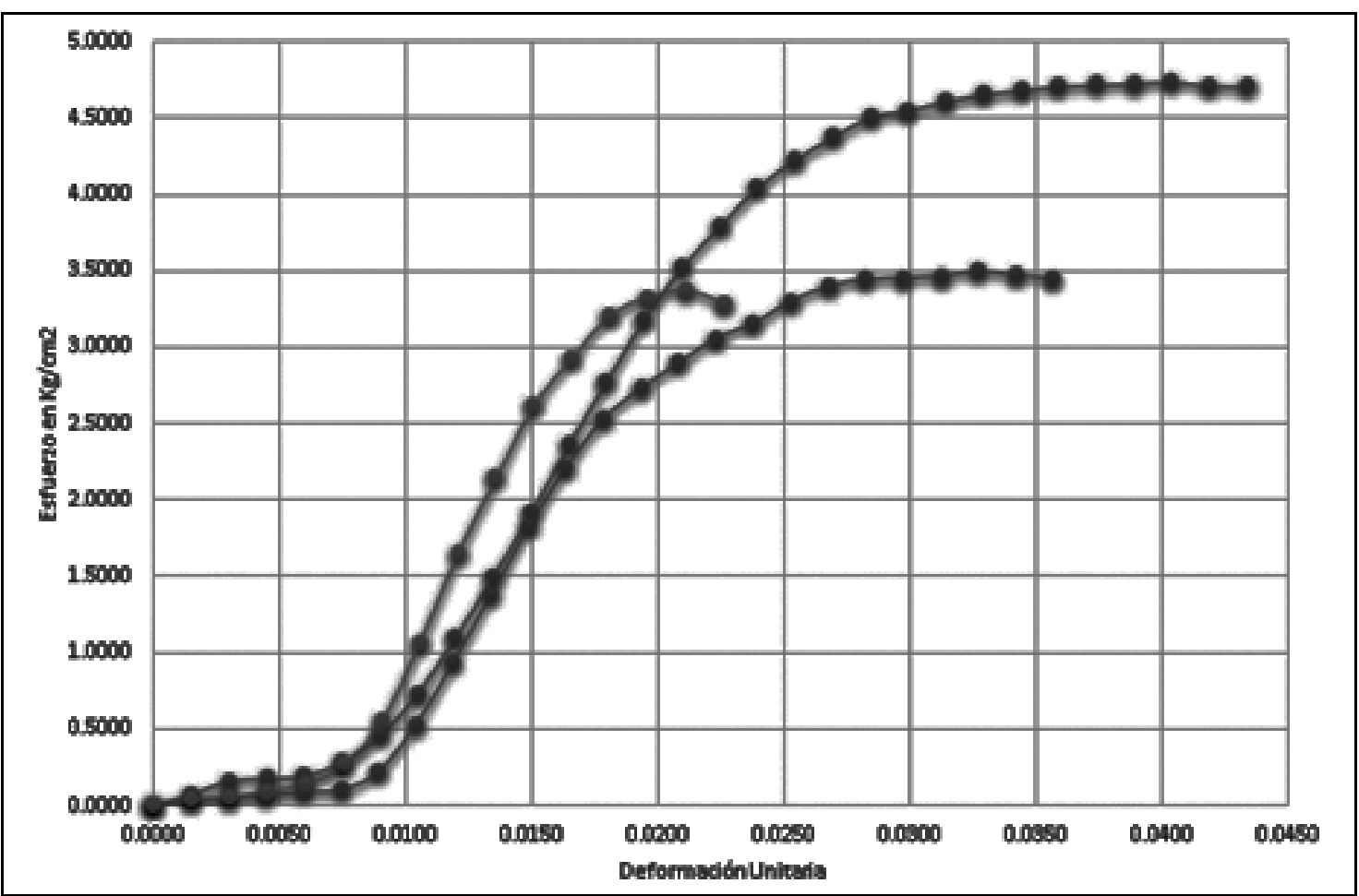

Figura 4. Gráfica esfuerzo- Deformación unitaria de arcilla con Cal. 


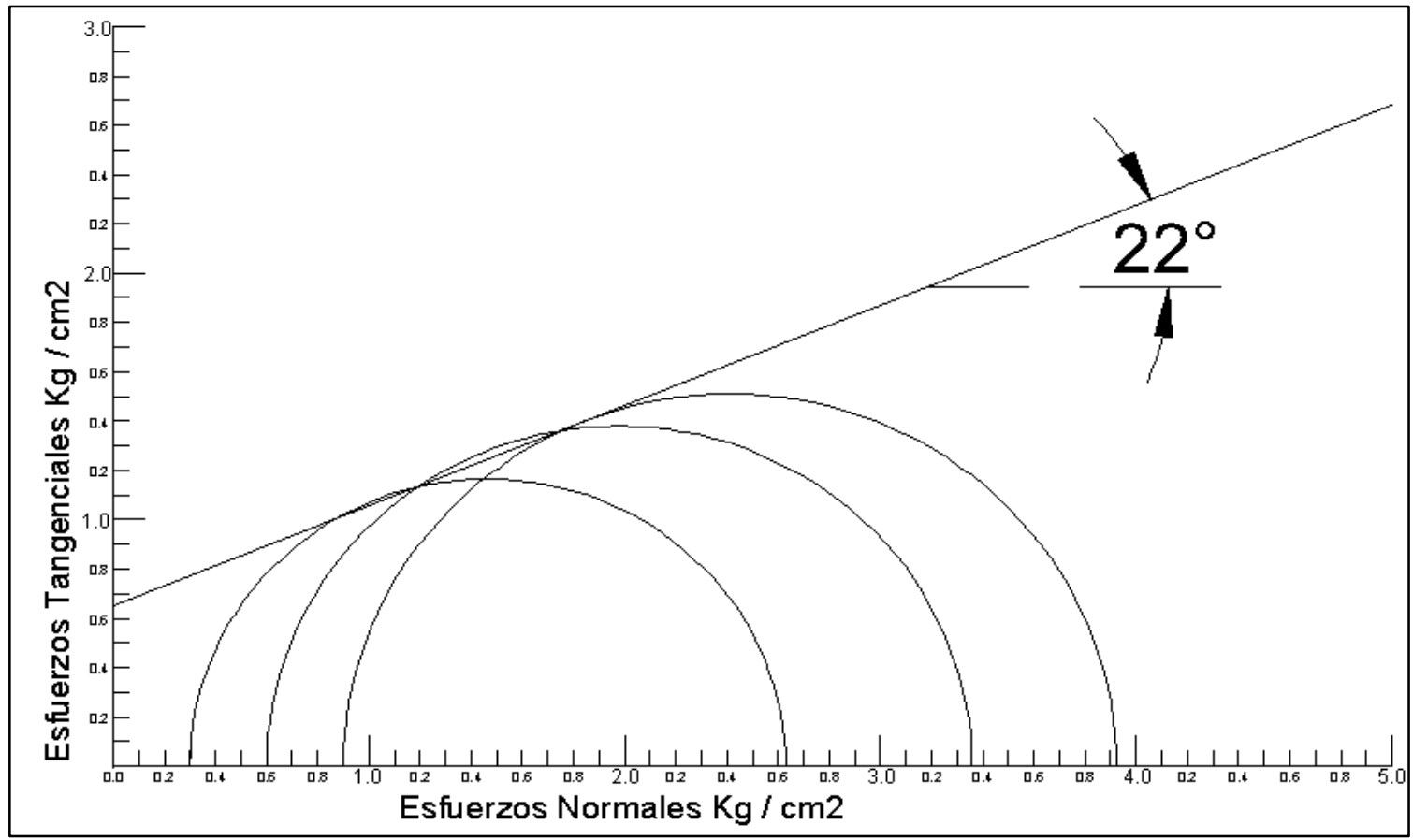

Figura 5. Círculos de Mohr de arcilla testigo de Santiago Undameo.

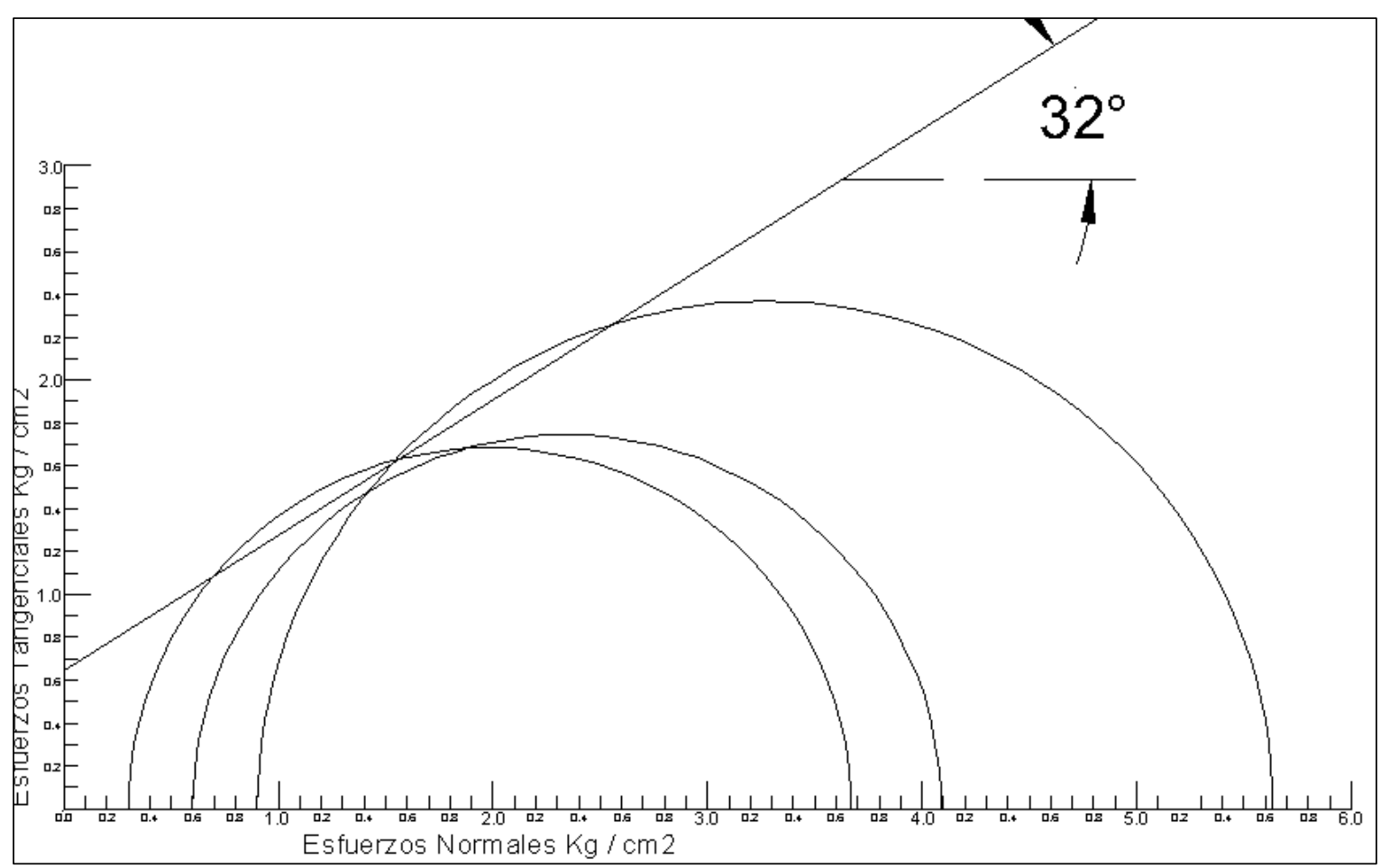

Figura 6. Círculos de Mohr de arcilla con Cal.

Tabla 2. Resultados de la envolvente de falla de las pruebas triaxiales UU.

\begin{tabular}{|c|c|c|}
\hline Aditivo & Cohesión $\left(\mathrm{kg} / \mathrm{cm}^{2}\right)$ & Ángulo de fricción $\left(^{\circ}\right)$ \\
\hline Arcilla Testigo & 0.6534 & 22 \\
\hline Arcilla con Cal & 0.7410 & 32 \\
\hline
\end{tabular}




\section{LIMITACIONES}

Los resultados obtenidos son solo aplicables para la arcilla de Santiago Undameo, México. Esto debido a que las arcillas tienes diferentes propiedades por lo tanto presentara diferente comportamiento en sus propiedades físicas y mecánicas en otro tipo de suelo.

\section{CONCLUSIONES}

Para cada una de las muestras en estudio se realizó una gráfica de esfuerzo contra deformación unitaria para observar el comportamiento que sufre la muestra cuando se realiza la aplicación de una carga Figura 3 y Figura 4, de las cuales se observa que la arcilla con Cal tiene mayor resistencia a la carga que se aplica.

En la Figura 7 se puede observar el incremento en la resistencia al corte dado por la envolvente de falla de la cual si es mayor resiste mejor a los esfuerzos, por lo tanto, la arcilla con cemento Portland tiene una mayor envolvente de falla, lo que nos indica que hubo un mejoramiento en sus propiedades mecánicas.

\section{REFERENCIAS}

Braja M. Das. (2001). Fundamento de ingeniería geotécnica. California. Editorial Thomsom Learning. Juárez Badillo y Rico Rodríguez. (2005). Mecánica de suelos Tomo 1 Fundamentos de la mecánica de suelos. 3era Edición. Editorial Limusa.

Miranda Leal Omar Salvador. (2017). Envolvente de falla en arcilla de Santiago Undameo, con diferentes estabilizadores; realizados en la maquina triaxial Soiltest $t$-500. Tesis de Licenciatura UMSNH. p 179, Facultad de Ingeniería Civil, UMSNH, México: Morelia. 